\title{
THE LOGARITHMIC LIMIT-SET OF AN ALGEBRAIC VARIETY
}

BY

\author{
GEORGE M. BERGMAN( $\left.{ }^{1}\right)$
}

\begin{abstract}
Let $C$ be the field of complex numbers and $V$ a subvariety of $(C-\{0\})^{n}$. To study the "exponential behavior of $V$ at infinity", we define $V_{\infty}^{(a)}$ as the set of limitpoints on the unit sphere $S^{n-1}$ of the set of real $n$-tuples $\left(u_{x} \log \left|x_{1}\right|, \ldots, u_{x} \log \left|x_{n}\right|\right)$, where $x \in V$ and $u_{x}=\left(1+\sum\left(\log \left|x_{i}\right|\right)^{2}\right)^{-1 / 2}$. More algebraically, in the case of arbitrary base-field $k$ we can look at places "at infinity" on $V$ and use the values of the associated valuations on $X_{1}, \ldots, X_{n}$ to construct an analogous set $V_{\infty}^{(b)}$. Thirdly, simply by studying the terms occurring in elements of the ideal $I$ defining $V$, we define another closely related set, $V_{\infty}^{(c)}$.

These concepts are introduced to prove a conjecture of A. E. Zalessky on the action of $G L(n, Z)$ on $k\left[X_{1}^{ \pm 1}, \ldots, X_{n}^{ \pm 1}\right]$, then studied further.

It is shown among other things that $V_{\infty}^{(b)}=V_{\infty}^{(c)} \supseteq$ (when defined) $V_{\infty}^{(a)}$. If a certain natural conjecture is true, then equality holds where we wrote " $\supseteq$ ", and the common set $V_{\infty} \subseteq S^{n-1}$ is a finite union of convex spherical polytopes.
\end{abstract}

1. A conjecture of Zalessky. Let $k$ be a field, and $k\left[X^{ \pm}\right]=k\left[X_{1}^{ \pm 1}, \ldots, X_{n}^{ \pm 1}\right]$ the ring obtained by adjoining $n$ commuting indeterminates and their inverses to $k$. This is the group algebra on the free abelian group of $\operatorname{rank} n, Z^{n}$, so $G L(n, Z)$ has a natural action on it.

Call a subgroup of $Z^{n}$ nontrivial if it is of infinite order and infinite index in $Z^{n}$; and call an ideal $I \subseteq k\left[X^{ \pm}\right]$nontrivial if it is of infinite dimension (i.e., nonzero) and infinite codimension in $k\left[X^{ \pm}\right]$as $k$-vector spaces. A. E. Zalessky conjectures in [1, Problem V.9], and we shall here prove:

THEOREM 1. Let I be a nontrivial ideal in $k\left[X^{ \pm}\right]$, and $H \subseteq G L(n, Z)$ the stabilizer subgroup of $I$. Then $H$ has a subgroup $H_{0}$ of finite index, which stabilizes a nontrivial subgroup of $Z^{n}$ (equivalently, which can be put into block-triangular form

$$
\left(\frac{\left.\right|^{*}}{0||^{*}}\right)
$$

in $G L(n, Z))$.

Received by the editors February 10, 1970 and, in revised form, April 10, 1970.

AMS 1970 subject classifications. Primary 14X00, 14A99, 14C99, 10M05; Secondary 10E05, 10M20, 12J05, 12J20, 13C15, 20G30.

Key words and phrases. Variety, exponential behavior at infinity, valuation, place, convex polytope, general linear group, dimension, logarithm, absolute value, sphere at infinity.

(1) Part of this work was done while the author was supported by a Science Research Council fellowship. 
The conclusion is equivalent to saying that some nontrivial subgroup of $\boldsymbol{Z}^{n}$ has finite orbit under the action of $H$, and hence to the statement that $H$ stabilizes some finite family of nontrivial subgroups of $Z^{n}$. We shall prove it in this last form.

The proof is sketched conceptually for $k=C$, the complex numbers, in $\S 2$. In $\S 3$ a version of the proof valid for any $k$ is given.

In the remaining $\S \S 4-8$, we shall formulate precisely and study further the basic concept used in these proofs: the set of "logarithmic points at infinity" of a variety.

(Note. An ordinary polynomial ring $k[X]=k\left[X_{1}, \ldots, X_{n}\right]$ can be looked at as the symmetric tensor algebra on the $n$-dimensional $k$-vector space $k^{n}$, and so a natural action of the automorphism group of this space, $\boldsymbol{G L}(n, k)$ on $k[X]$ is induced. The analog of Zalessky's conjecture for this action is not true. E.g., the stabilizer of the principal ideal generated by $\left(\sum X_{i}^{2}\right)-1$ is the orthogonal group $\boldsymbol{O}(n, k)$, but this stabilizes no finite family of subspaces of $k^{n}$, when $n \geqq 3$ and $k$ is infinite.)

2. Assume $k=C$, and let $V \subseteq(C-\{0\})^{n}$ be the variety defined by a nontrivial ideal $I$. We shall consider the behavior of $V$ "near infinity". Usually, this means looking at the limiting values of the coordinate-ratios $x_{1}: x_{2}: \ldots: x_{n}$ as (at least some of) the coordinates of $x \in V$ becomes large. But because of the way $G L(n, Z)$ acts here, it is more natural for us to consider the ratios $\log \left|x_{1}\right|: \cdots: \log \left|x_{n}\right|\left(^{2}\right)$. We shall understand the "ratio" of such a sequence of real numbers (not all zero) to mean its equivalence class under multiplication by positive real constants, so that the set of all ratios can be identified with the $n-1$-sphere $S^{n-1}$, rather than projective space.

The set of limits of coordinate-ratios $x_{1}: \cdots: x_{n}(x \in V, V$ a nontrivial variety in $C^{n}$ ) as $x$ becomes large can form an arbitrary proper nonempty subvariety of complex projective $(n-1)$-space. But the limiting ratios of the logarithms $\left({ }^{3}\right)$ will form a different sort of set. In particular, we shall soon see that it lies in a finite union of proper great subspheres on $S^{n-1}$, having rational defining parameters.

Assuming this for the moment, we note that the intersection of two such finite unions of subspheres will again be one, and the family of all finite unions of great subspheres is easily seen to have descending chain condition. Consequently, there will exist a unique finite union $U$ of subspheres minimal for the property of containing all "logarithmic limit-points at infinity" of $V$. Further, $V$ is of positive dimension, hence is unbounded, hence its set of logarithmic limit-points on $S^{n-1}$ will be nonempty, so $U$ must be nonempty.

Now the space $\boldsymbol{R}^{n}$, in which we took our $n$-tuples of logarithms, arises as the dual of $Z^{n}$, that is, $\operatorname{Hom}_{\text {groups }}\left(Z^{n}, \boldsymbol{R}\right)$. Thus we get a natural action of $G L(n, Z)$ on $\boldsymbol{R}^{n}$, and so on $\boldsymbol{S}^{n-1}$. Clearly, $U$ will be invariant under the induced action of the

$\left.{ }^{2}\right)$ D. Mumford has pointed out to me that this point of view is similar to that of the theory of Néron models-cf. [4].

$\left({ }^{3}\right)$ For a formal definition, cf. the abstract, or $\$ 5$ below. 
stabilizer subgroup, $H$, of $I$. By duality, we can obtain from the great subspheres of $U$ a family $Q$ of nontrivial subgroups of $Z^{n}$, also invariant under $H$. Q.E.D.

To establish the claim that the logarithmic points at infinity of $V$ lie in a finite union of proper great subspheres of $S^{n-1}$, choose any nonzero $a \in I$, and define the support of $a$ to be the set $s(a)$ of $\alpha \in Z^{n}$ such that $X^{\alpha}$ occurs with nonzero coefficient in $a$. This will be finite, say of cardinality $r$. We now write $a=\sum c_{\alpha} X^{\alpha}\left(\alpha \in s(a), c_{\alpha} \in C-\{0\}\right)$. At each point $x=\left(x_{1}, \ldots, x_{n}\right) \in V$ we have $\sum c_{\alpha} x^{\alpha}=0$. Hence, at each point two terms of this sum-the two of largest absolute value, for instance-must be of the same order of magnitude. Precisely, there will exist $\alpha \neq \beta \in s(a)$ such that $1 /(r-1) \leqq\left|c_{\alpha} X^{\alpha} / c_{\beta} x^{\beta}\right| \leqq r-1$.

Taking logarithms, and putting $C=\sup _{\alpha, \beta \in s(a)} \log (r-1)\left|c_{\beta} / c_{\alpha}\right|$, we get:

$$
\forall x \in V \exists \alpha, \beta \in s(a), \alpha \neq \beta:-C \leqq \log \left|x^{\alpha-\beta}\right| \leqq C .
$$

If we rewrite $\log \left|x^{\alpha-\beta}\right|$ as $(\alpha-\beta) \cdot \log |x|$ (dot-product of vectors!), we see that this says that each $n$-tuple $\log |x|=\left(\log \left|x_{1}\right|, \ldots, \log \left|x_{n}\right|\right)(x \in V)$ lies in one of the finite family of "planks" in $\boldsymbol{R}^{n}$ (one for each pair $\alpha \neq \beta \in s(a)$ ) defined by the linear inequalities $-C \leqq(\alpha-\beta) \cdot \log |x| \leqq C$. Clearly this restricts the "points at infinity" to a corresponding finite family of great subspheres of $S^{n-1}$.

3. In this section $k$ will be an arbitrary field, and $V$ will denote $\operatorname{Spec} k\left[X^{ \pm}\right] / I$; however, we shall refer to $V$ only to give "geometric" interpretations to operations we will describe algebraically. We shall use the same symbols $X_{1}, \ldots, X_{n}$ to designate elements of $k\left[X^{ \pm}\right]$and their images in $k\left[X^{ \pm}\right] / I$.

The analog of "letting point-coordinates go to infinity" will here be to look at "places at infinity" on the variety $V$. Since the usual definition of a place requires an integral domain, let us choose an arbitrary prime ideal $\mathfrak{p} \supseteq I$, still of infinite codimension in $k\left[X^{ \pm}\right]$-i.e., we choose a reduced irreducible subvariety of $V$, of positive dimension. Then by [5, Chapter VI, Theorem 6], the field of fractions of $k\left[X^{ \pm}\right] / \mathfrak{p}$ will have a place whose valuation ring does not contain all of $k\left[X^{ \pm}\right] / \mathfrak{p}$. This means we can find a function (the associated valuation, restricted to our ring) $v: k\left[X^{ \pm}\right] / \mathfrak{p} \rightarrow A \cup\{+\infty\}$, where $A$ is a totally ordered abelian group, with the properties:

(1) $v(a)=0$ for $a \in k-\{0\} ; v(0)=+\infty$,

(2) $v(a b)=v(a)+v(b)$

(3) $v(a-b) \geqq \inf (v(a), v(b))$,

(4) $v(a)=+\infty \Rightarrow a=0$, and

(5) $v$ assumes a negative value on some element of the ring.

This induces a function (which we shall by abuse of symbolism again call $v$ ) on $k\left[X^{ \pm}\right] / I$ satisfying all these properties except for (4), which we do not need. We shall call such a function a place embedded in $V$ at infinity. Note that (5) tells us that the homomorphism of $Z^{n}$ into $A$ given by $\alpha \mapsto v\left(X^{\alpha}\right)$ is nonzero-since all elements of our ring are linear combinations of the $X^{\alpha}$. 
We shall say that $v$ annihilates a subgroup $G \subseteq Z^{n}$ if $v\left(X^{\alpha}\right)=0$ for all $\alpha \in G$.

Let a subgroup system mean a finite nonempty family $S$ of subgroups of $Z^{n}$. We shall write $S \geqq T$, for subgroup systems $S$ and $T$, if every member of $S$ contains some member of $T$, and call $S$ and $T$ equivalent if $S \geqq T$ and $T \geqq S$ (easily seen to be an equivalence relation). $S$ will be called irredundant if no subgroup belonging to $S$ properly contains another. It is easy to see that any subgroup system $S$ is equivalent to a unique irredundant subgroup system $S^{\prime}$, consisting of the minimal subgroups in $S$.

A subgroup system $S$ will be called compatible with $V$ if every place embedded in $V$ at infinity annihilates some subgroup belonging to $S$. Note that if $S \geqq T$ and $S$ is compatible with $V$, then so is $T$. (Hence if $S$ and $T$ are equivalent and one is compatible with $V$, so is the other.)

The equivalence classes of subgroup systems will form a lattice, with least upper bound given by $S \vee T=\{G+H \mid G \in S, H \in T\}$, and greatest lower bound given by set-theoretic union. It is easy to verify that this lattice will have ascending chain condition. (If $S$ is irredundant, let $p_{i}(S)$ denote the number of groups of rank $i$ in $S$. Then for $T>S$, we have $\left(p_{1}(T), \ldots, p_{n}(T)\right)<\left(p_{1}(S), \ldots, p_{n}(S)\right)$ under lexicographic ordering.) The lattice also has a minimal element, $\{\{0\}\}$, and a maximal element, $\left\{\boldsymbol{Z}^{n}\right\}$.

It is easy to verify, from our construction for $S \vee T$, that if $S$ and $T$ are compatible with $V$, so is $S \vee T$. It follows that there will exist a unique maximal irredundant subgroup system compatible with $V$. Let us call this $Q$.

Now let us choose any nonzero element $a=\sum_{s(a)} c_{\alpha} X^{\alpha} \in I\left(c_{\alpha} \in k-\{0\}\right)$. For any place $v$ embedded in $V$ at infinity, $v\left(\sum c_{\alpha} X^{\alpha}\right)=v(0)=+\infty$, hence two of the terms $v\left(X^{\alpha}\right)$ and $v\left(X^{\beta}\right)(\alpha, \beta \in s(a))$ must be equal. (In fact, the minimum value of $v\left(X^{\alpha}\right)$ among the $\alpha$ 's in $s(a)$ must be assumed twice.) Thus $v\left(X^{\alpha-\beta}\right)=0$. Thus, every such $v$ annihilates a cyclic subgroup of $\boldsymbol{Z}^{n}$ generated by one of the differences $\alpha-\beta$ $(\alpha, \beta \in s(a), \alpha \neq \beta)$, which means that the subgroup system $S$ consisting of this finite family of cyclic groups is compatible with $V$. Hence our maximal subgroup system $Q$ compatible with $V$ is $>\{\{0\}\}$. Also, $Q<\left\{\boldsymbol{Z}^{n}\right\}$, for we showed earlier that we can construct at least one place embedded in $V$ at infinity, and this will not annihilate all of $Z^{n}$.

It follows that $Q$ is a finite nonempty family of nontrivial subgroups of $Z^{n}$, and it will clearly be invariant under $H$, the stabilizer of $I$, which completes the proof of Zalessky's conjecture for arbitrary base-field.

4. In the next section we shall formalize the ideas used in the above proofs, but we wish to make some preliminary observations.

When we studied "points at infinity" as represented by places, we allowed these to be defined by maps into an arbitrary ordered abelian group. Now if $u$ is a positive element of an ordered abelian group $A$, the set $\{t \in A \mid \exists m:-m u<t<m u\}$ forms a subgroup $A_{u} \subseteq A$, which has a nonzero homomorphism of ordered groups into the real numbers $\boldsymbol{R}$, uniquely determined by assigning a positive value to the 
image of $u$. The images of the other elements are then determined by the relation $m t \geqq m^{\prime} u$ and $m t \leqq m^{\prime} u$ which they satisfy. (This map may have nontrivial kernel.) Now if $v: k\left[X^{ \pm}\right] / I \rightarrow A \cup\{+\infty\}$ is a place embedded in $V$ at infinity, let $u$ be the maximum of the $v\left(X_{i}^{ \pm 1}\right)(i=1, \ldots, n)$. Note that every element $v(a)\left(a \in k\left[X^{ \pm}\right] / I\right)$ will be $\geqq m u$ for some $m \in Z$. Let us construct a map $\varphi: A_{u} \rightarrow \boldsymbol{R}$ as above. We can now define a real-valued place $v^{\prime}$ as follows: If $v(a) \in A_{u}$, let $v^{\prime}(a)=\varphi v(a)$. In the contrary case, $v(a)$ must be greater than every $m u$, and we define $v^{\prime}(a)=+\infty$. Note that $v^{\prime} \mid X Z^{n}$ is nonzero. Thus embedded places with real value-group-or realvalued valuations as we shall call them now-provide a good approximation to the most general valuation, and we shall limit our consideration to the former in what follows. To be precise, we shall understand by a valuation on an algebra a function into $\boldsymbol{R} \cup\{+\infty\}$ satisfying conditions (1)-(3) of $\S 3$, and the valuations we deal with will always satisfy a further restriction implying condition (5).

With our valuations all real-valued, we will be able to obtain from them points of $\boldsymbol{S}^{n-1}$, giving a treatment parallel to that which we had using logarithms, rather than having to work with "dual" objects: subgroups of $\boldsymbol{Z}^{n}$. We shall also define $S^{n-1}$ to be, concretely, the unit sphere $\left\{p \in \boldsymbol{R}^{n} \mid \sum p_{i}^{2}=1\right\}$.

Let us now look at a very simple example of these "sets at infinity". Let $V$ be the subset of $(C-\{0\})^{2}$ defined by an equation $x_{1}+x_{2}=c(c \neq 0)$. In $V, \log \left|x_{1}\right|$ and $\log \left|x_{2}\right|$ can approach $+\infty$ in the ratio $1: 1$, or $\log \left|x_{1}\right|$ can approach $-\infty$ while $\log \left|x_{2}\right|$ remains bounded, or vice versa. Projecting these three directions onto the unit circle $S^{1}$, we get three points $\left(2^{-1 / 2}, 2^{-1 / 2}\right),(-1,0)$ and $(0,-1)$.

When we use valuations, we must reverse signs because a "large" element is one with a negative valuation. If $v$ is a valuation on a ring $k\left[X_{1}^{ \pm 1}, X_{2}^{ \pm 1}\right] /\left(X_{1}+X_{2}-c\right)$, which is nonzero on at least one of $X_{1}$ and $X_{2}$, then either $v\left(X_{1}\right)=v\left(X_{2}\right)<0$, or $v\left(X_{1}\right)>0=v\left(X_{2}\right)$, or vice versa. Reversing signs and projecting on $S^{1}$, we again get the points $\left(2^{-1 / 2}, 2^{-1 / 2}\right),(-1,0)$ and $(0,-1)$.

If one analyzes similarly the equation in three variables, $X_{1}+X_{2}+X_{3}=c$, one finds that the induced subset of $S^{2}$ consists of 6 arcs of great circles, which form the 2-skeleton of a tetrahedron!

5. Formal definitions. Let $k$ be a field, $n$ a positive integer, $k\left[X^{ \pm}\right]$as previously defined, $I$ any ideal of this ring, and $V$ the associated subvariety of Spec $k\left[X^{ \pm}\right]$.

By an "absolute value" on $k$, we shall understand a function $\mid$ into nonnegative real numbers, satisfying $|0|=0,|1|=1,|x y|=|x||y|,|x+y| \leqq|x|+|y|$, and $\exists x, 0<|x|<1$.

(a) Suppose $k$ has an absolute value function | $\mid$. Then we can map the set of $k$-valued points of $V$, a subset of $(k-\{0\})^{n}$, into the interior of the real closed unit ball $\boldsymbol{B}^{n}$ by sending $\left(x_{1}, \ldots, x_{n}\right)$ to $\left(u_{x} \log \left|x_{1}\right|, \ldots, u_{x} \log \left|x_{n}\right|\right)$, where $u_{x}$ $=\left(1+\sum\left(\log \left|x_{j}\right|^{2}\right)\right)^{-1 / 2}$. We define $V_{\infty}^{(a)}$ as the intersection of the closure of this image with the boundary $S^{n-1}$ of the unit ball. In this construction, we will want $k$ algebraically closed.

(b) Define $V_{\infty}^{(b)}$ as the set of $n$-tuples $\left(-v\left(X_{1}\right), \ldots,-v\left(X_{n}\right)\right)$ as $v$ runs over all 
real-valued valuations on $k\left[X^{ \pm}\right] / I$ satisfying $\sum v\left(X_{j}\right)^{2}=1$. (These will satisfy condition (5) of $\S 3$ because not all $v\left(X_{j}\right)$ can be 0 .)

(c) As before, we define the support $s(a)$ of an element $a \in k\left[X^{ \pm}\right]$to be the set of $\alpha \in Z^{n}$ such that $X^{\alpha}$ occurs with nonzero coefficient in $a$. We define $V_{\infty}^{(c)}$ to be the set of points $\xi \in S^{n-1}$ such that for all nonzero $a \in I$, the maximum value of the dot product $\xi \cdot \alpha$ as $\alpha$ runs over $s(a)$ is assumed at least twice.

We can now state the key properties of "points at infinity" used in our proof of Zalessky's conjecture as:

(i) If $V$ is of positive dimension then $V_{\infty}^{(a)}$ (respectively, $V_{\infty}^{(b)}$ ) is nonempty.

(ii) If $I$ is nonzero, then $V_{\infty}^{(c)}$ lies in a finite union of proper subspheres of $S^{n-1}$, defined over the rationals.

(iii) $V_{\infty}^{(c)}$ contains $V_{\infty}^{(a)}$ and $V_{\infty}^{(b)}$.

In the next three sections we shall prove much stronger versions of each of these observations. Let us make one more elementary observation here.

Lemma. If $V$ is a subvariety of Spec $k\left[X^{ \pm}\right]$, then $V_{\infty}^{(a)}$ (resp. $V_{\infty}^{(b)}$ ) is the union, over the reduced irreducible components $U \subseteq V$, of the sets $U_{\infty}^{(a)}$ (resp. $\left.U_{\infty}^{(b)}\right)$.

Proof. This is immediate for $V_{\infty}^{(a)}$. To see it for $V_{\infty}^{(b)}$, let $I$ be the ideal associated with $V$. A valuation on $k\left[X^{ \pm}\right] / I$ is equivalent to a valuation $v$ on $k\left[X^{ \pm}\right]$such that the set $v^{-1}(+\infty)$-which is always a prime ideal-contains $I$. Clearly $v^{-1}(+\infty)$ contains $I$ if and only if it contains one of the minimal prime ideals containing $I$, and these are the ideals corresponding to the reduced irreducible components of $V$.

6. The relation between $V_{\infty}^{(a)}, V_{\infty}^{(b)}$, and $V_{\infty}^{(c)}$. We can strengthen observation (iii) to

THEOREM 2. Let $k\left[X^{ \pm}\right]$and I be as above. Then $V_{\infty}^{(b)}=V_{\infty}^{(c)}$.

Also, if $k$ is algebraically closed and given with an absolute value function, then $V_{\infty}^{(a)}$ is a closed subset of $V_{\infty}^{(b)}=V_{\infty}^{(c)}$, containing all points thereof with rational coordinate-ratios.

Proof. We shall show (1) $V_{\infty}^{(b)} \subseteq V_{\infty}^{(c)}$, (2) $V_{\infty}^{(c)} \supseteq V_{\infty}^{(b)}$, and, assuming an absolute value function given on $k$, (3) $V_{\infty}^{(a)} \subseteq V_{\infty}^{(c)}$, and finally, assuming $k$ is also algebraically closed $\left(V_{\infty}^{(c)}\right)_{\text {rat. }} \subseteq V_{\infty}^{(a)}$. That $V_{\infty}^{(a)}$ is closed in $S^{n-1}$ is clear from the definition.

(1) Consider any element $\xi=\left(-v\left(X_{1}\right), \ldots,-v\left(X_{n}\right)\right) \in V_{\infty}^{(b)}, v$ a valuation on $k\left[X^{ \pm}\right] / I$. For any nonzero $a=\sum c_{\alpha} X^{\alpha} \in I$, we note that $v(a)=v(0)=+\infty$, so the minimum value of the terms $v\left(X^{\alpha}\right)=-\xi \cdot \alpha(\alpha \in s(a))$ must be assumed twice, whence $\xi \in V_{\infty}^{(c)}$.

(2) Let $\xi \in V_{\infty}^{(c)}$. Define a valuation $v$ on $k\left[X^{ \pm}\right]$by $v(a)=\inf _{\alpha \in s(a)}-\xi \cdot \alpha$. If we designate by $S$ the multiplicative group generated by $X_{1}, \ldots, X_{n} \in k\left[X^{ \pm}\right]$, then the hypothesis $\xi \in V_{\infty}^{(c)}$ tells us that no member of $I$ is equal to a single element of $S$ plus an element of higher valuation under $v$. Corollary 1 of [2] (and see also comment after Corollary 2) says that in this situation, there will exist a valuation 
$v^{\prime} \geqq v$ on $k\left[X^{ \pm}\right]$equal to $+\infty$ on all of $I$, and agreeing with $v$ on $S$. This is equivalent to a valuation $v^{\prime \prime}$ on $k\left[X^{ \pm}\right] / I$ satisfying $\left(-v\left(X_{1}\right), \ldots,-v\left(X_{n}\right)\right)=\xi$. Q.E.D.

(3) Let $a=\sum c_{\alpha} X^{\alpha}$ be any nonzero element of $I, r$ the cardinality of $s(a)$, which we can assume $>1$ (otherwise $I=k\left[X^{ \pm}\right]$and the result is trivial), $\dot{m}=\min _{\alpha \in s(a)}\left|c_{\alpha}\right|$, and $M=\max _{\alpha \in s(a)}\left|c_{\alpha}\right|$. Given any family of real numbers $\left(t_{j}\right)$ indexed by a set $J$ of finite cardinality $>1$, let us write $\max _{J}^{(2)} t_{j}$ for the second largest value in our family, equal to $\max _{J} t$, if this value is assumed twice. This will be a continuous function of the $J$-tuple $\left(t_{j}\right)$.

Now as in $\S 2$, we see that for all $x \in V$,

$$
\left(\max _{\alpha \in S(a)}\left|c_{\alpha} x^{\alpha}\right|\right) /\left(\max _{\alpha \in s(a)}^{(2)}\left|c_{\alpha} x^{\alpha}\right|\right) \leqq r-1 .
$$

Since $\max \left|c_{\alpha} x^{\alpha}\right| \leqq M \max \left|x^{\alpha}\right|$, and $\max ^{(2)}\left|c_{\alpha} x^{\alpha}\right| \geqq m \max ^{(2)}\left|x^{\alpha}\right|$, we get

$$
\left(\max _{\alpha \in s(a)}\left|x^{\alpha}\right|\right) /\left(\max _{\alpha \in s(a)}^{(2)}\left|x^{\alpha}\right|\right) \leqq M(r-1) / m .
$$

Taking logarithms of both sides, and multiplying by the function $u_{x}$ in the definition of $V_{\infty}^{(a)}$, we get

$$
\max _{\alpha \in s(a)}\left(\alpha \cdot u_{x} \log |x|\right)-\max _{\alpha \in s(a)}^{(2)}\left(\alpha \cdot u_{x} \log |x|\right) \leqq u_{x} \log (M(r-1) / m) .
$$

Now let $x$ vary so that $u_{x} \log |x|$ approaches an arbitrary point $\xi$ of $V_{\infty}^{(a)}$. Then $u_{x}$ will approach 0 , hence so will the right-hand side of the above equation, and taking the limit, we get

$$
\max _{\alpha \in s(a)} \alpha \cdot \xi-\max _{\alpha \in S(a)}^{(2)} \alpha \cdot \xi=0
$$

i.e., $\xi \in V_{\infty}^{(c)}$. Q.E.D.

Let us indicate another approach, by which one can prove that $V_{\infty}^{(a)} \subseteq V_{\infty}^{(b)}$. One associates to every $x \in V$ the function $p_{x}: a \mapsto-u_{x} \log a(x)$, and considers $p_{x}$ a point of the compact space $[-\infty,+\infty]^{k\left[X^{ \pm}\right] / I}$. Given a filter $\mathscr{F}$ in $V$ such that $\lim _{\mathscr{F}} u_{x} \log |x|$ is an arbitrary point $\xi$ of $V_{\infty}^{(a)}$, let $v \in[-\infty,+\infty]^{k\left[X^{ \pm}\right] / I}$ be a cluster point of the $p_{x}$ under $\mathscr{F}$. Then it is not hard to show that $v$ is $(-\infty,+\infty]$-valued, and is in fact a valuation on $k\left[X^{ \pm}\right] / I$ such that $\left(-v\left(X_{1}\right), \ldots,-v\left(X_{n}\right)\right)=\xi$.

(4) Let $\xi$ be a point of $V_{\infty}^{(c)}$ the ratios of whose coordinates are rational; we wish to prove $\xi \in V_{\infty}^{(a)}$. Making use of the action of $G L(n, Z)$ on $k\left[X^{ \pm}\right]$(cf. $\left.\S 1\right)$ and the induced action on $S^{n-1}$ (not isometric!), we can reduce to the case where $\xi=(0, \ldots, 0,-1)$.

Let $R^{\prime}$ designate the ring $k\left[X_{1}^{ \pm 1}, \ldots, X_{n-1}^{ \pm 1} ; X_{n}\right] \subseteq R=k\left[X^{ \pm}\right]$, and $R_{0}$ the ring $k\left[X_{1}^{ \pm 1}, \ldots, X_{n-1}^{ \pm 1}\right]$, into which we map $R^{\prime}$ by sending $X_{n}$ to 0 . Let $I^{\prime}$ designate $I \cap R^{\prime}$, and $I_{0}$ the image of $I^{\prime}$ in $R_{0}$. Thus, $I_{0}$ consists of the components of degree 0 in $X_{n}$ of elements of $I^{\prime}$. Let $V^{\prime}$ designate the subvariety of $(k-\{0\})^{n-1} \times k$ defined by $I^{\prime}$, and $V_{0}=V^{\prime} \cap\left((k-\{0\})^{n-1} \times\{0\}\right)$, the subvariety of $(k-\{0\})^{n-1} \times\{0\}$ defined by $I_{0} . V^{\prime}$ will be the Zariski closure of $V$ in $(k-\{0\})^{n-1} \times k$, and $V=V^{\prime}-V_{0}$. 
If $(0, \ldots, 0,-1) \in V_{\infty}^{(c)}$, this means that every nonzero $a \in I$ has more than one term of minimal degree in $X_{n}$. Hence every nonzero element of $I_{0}$ has more than one term, i.e., $I_{0}$ is a proper ideal of $R_{0}$. Then $V_{0}$ is nonempty: let $x \in V_{0}$.

Now $V$ will in fact be dense in $V^{\prime}$ under the topology induced by the absolute value on $k$. For $k=$ the complex numbers, this comes from [3, Theorem I.10.1, p. 111]; we show in the appendix to this article that the proof can be modified to work for any algebraically closed field topologized by a nontrivial absolute value. Hence we can obtain $x$ as a limit of points $y \in V$. As $y$ approaches $x, \log \left|y_{i}\right|$ will approach the finite values $\log \left|x_{i}\right|$ for $i=1, \ldots, n-1$, but $\log \left|y_{n}\right|$ will approach $-\infty$. Hence the points $u_{y} \log |y| \in B^{n}$ (see definition of $\left.V_{\infty}^{(a)}\right)$ will approach $(0, \ldots, 0,-1)$. Hence that point lies in $V_{\infty}^{(a)}$. Q.E.D.

Henceforth, we shall write $V_{\infty}$ for $V_{\infty}^{(b)}=V_{\infty}^{(c)}$.

A conjecture we shall make in the next section, which seems very likely to be true, would imply that points with rational coordinate-ratios are dense in $V_{\infty}$, and hence that for $k$ an algebraically closed field with absolute value, $V_{\infty}^{(a)}$ also equals $V_{\infty}$. Another approach to proving this might be to attempt to prove a general statement such as that any real-valued valuation on a Noetherian ring is approximable by rational-valued valuations.

7. Duals of convex polytopes, and the geometric structure of $V_{\infty}$. Our definition of $V_{\infty}^{(c)}$ is related to an interesting geometric construction:

Given any bounded convex polytope $C$ in $R^{n}$ (i.e., polygon if $n=2$, polyhedron if $n=3$, etc.) containing a neighborhood of 0 , we may define the dual polytope $C^{*}$ as the set of $\xi \in \boldsymbol{R}^{n}$ such that $\sup _{\alpha \in C} \alpha \cdot \xi \leqq 1$. The boundary (n-1-skeleton) of $C^{*}$ will consist of those points $\xi$ for which this supremum is exactly 1 . To each vertex $\alpha_{0}$ of $C$ will correspond an $n-1$-face of $C^{*}$, the set of $\xi$ for which this value 1 is achieved at $\alpha_{0}$. Similarly, for each edge of $C$, we get an $n-2$-cell, the set for which the maximum value occurs all along this edge, etc.

The projection of the $n-2$-skeleton of $C^{*}$ on the unit sphere $S^{n-1}$ can be characterized as the set of $\xi$ of norm 1 such that the supremum $\sup _{\alpha \in C} \alpha \cdot \xi$ is achieved for more than one $\alpha$. This construction is invariant under translation of $C$ (which that of $C^{*}$ itself certainly is not); hence we shall now drop the restriction that $C$ contain a neighborhood of the origin, and we shall not even require that $C$ have nonempty interior in $\boldsymbol{R}^{n}$. We shall call this set the spherical dual of the polytope. We now see that given an ideal $I \subseteq k\left[X^{ \pm}\right]$, the set $V_{\infty}$ we have associated with $I$ is the intersection of the spherical duals of the convex hulls in $R^{n}$ of the sets $s(a)$, as $a$ runs over $I-\{0\}$.

Let us call a closed subset of $\boldsymbol{S}^{n-1}$ convex if it is the set of all $\xi$ satisfying a family of inequalities $\alpha \cdot \xi \geqq 0$, as $\alpha$ runs over some family $A \subseteq R^{n}$; let us call it a convex spherical polytope if $A$ can be taken finite, and let us say such a polytope is rational if $A$ can be taken to be a finite subset of $Z^{n}$. (Note that under this definition, a great subsphere of $S^{n-1}$ is a convex spherical polytope-e.g., an $n$-2-dimensional great subsphere is defined by one equation $\alpha \cdot \xi=0$, which can be written $\alpha \cdot \xi \geqq 0$, 
$\alpha \cdot(-\xi) \geqq 0$.) Then we assert that the spherical dual of the convex hull of a finite subset $A \subseteq Z^{n}$ of cardinality $r$ is a finite union of rational convex spherical polytopes. Indeed, it can be described as the union, over all $\alpha_{0}, \alpha_{1} \in A$ of the set of $\xi$ satisfying the $2 r$ inequalities $\left(\alpha_{0}-\alpha\right) \cdot \xi \geqq 0,\left(\alpha_{1}-\alpha\right) \cdot \xi \geqq 0$ where $\alpha$ ranges over $A$. Note also that a finite intersection of rational spherical polytopes is again one, and that in a rational spherical polytope, the set of points with rational coordinateratios is dense. (This is not true of general convex spherical polytopes. E.g., if $\alpha$ is an element of $\boldsymbol{R}^{n}$, whose coordinates are linearly independent over the rational numbers, then the great sphere given by $\alpha \cdot \xi=0$ contains no point with rational coordinate-ratios.)

If $I$ is a principal ideal of $k\left[X^{ \pm}\right]$, generated by an element $a_{1} \neq 0$, it is easy to show that the set $V_{\infty}$ is precisely the spherical dual of the convex hull of $s\left(a_{1}\right)$. This explains the tetrahedron we noted earlier. It is not true that if $a_{1}, \ldots, a_{m}$ generate $I$, then $V_{\infty}$ is the intersection of the spherical duals associated with these elements. For example, $X_{1}+X_{2}+1$ and $X_{1}+X_{2}+2$ yield the same three points of $S^{1}$, but generate the unit ideal, with $V_{\infty}=\varnothing$.

Conjecture. $V_{\infty}$ is always equal to the intersection of the spherical duals of the convex hulls of supports of a finite set of elements of $I$.

In particular, this would imply that $V_{\infty}$ is always a finite union of convex rational spherical polytopes, and hence that points of $V_{\infty}$ with rational coordinateratios are dense.

8. The dimension of $V_{\infty}$. By a rational great subsphere of $\boldsymbol{S}^{n-1}$ of dimension $n-m$, we shall mean the solution-set of a family of equations $\xi \cdot \alpha_{i}=0$ $(i=1, \ldots, m-1)$, where $\alpha_{1}, \ldots, \alpha_{m-1}$ are linearly independent elements of $Z^{n}$. A rational great hemisphere of dimension $n-m$ will mean the set defined by such a set of equations, and one inequality, $\xi \cdot \alpha_{m} \geqq 0$, where $\alpha_{1}, \ldots, \alpha_{m}$ are all linearly independent. The set of all $n-m$-dimensional great sub (hemi)spheres of $\boldsymbol{S}^{n-1}$ has a natural structure of compact manifold, in which the rational (hemi)spheres are dense, and such that the set of (hemi)spheres lying in a given open subset of $\boldsymbol{S}^{n-1}$ is open.

The first statement of the following theorem generalizes our observation that if $I \neq 0, V_{\infty}$ lies in a finite union of proper great subspheres of $S^{n-1}$; the second, the observation that if $V$ is of positive dimension, $V_{\infty} \neq \varnothing$.

THeOREM 3. Let $V$ be a reduced irreducible subvariety of Spec $k\left[X^{ \pm}\right]$, of dimension $m$. Then $V_{\infty}$ lies in a finite union of rational $m$-1-dimensional great subspheres of $S^{n-1}$, and has nonempty intersection with every $n-m$-dimensional great hemisphere.

Proof. Let $V$ be defined by the prime ideal $I \subseteq k\left[X^{ \pm}\right]$.

Proof of first assertion. Given any rational great subsphere $S_{0} \subseteq S^{n-1}$ of dimension $>m-1$, we shall construct a finite family $A$ of $n$-2-dimensional rational great subspheres of $S^{n-1}$ the union of which contains $V_{\infty}$, and no member of which 
contains $\boldsymbol{S}_{0}$. It is easily deduced that if we choose a minimal finite union of great subspheres which contains $V_{\infty}$, all the subspheres involved in this union must have dimension $\leqq m-1$.

Let $S_{0}$ be $m-1+r$-dimensional $(r \geqq 1)$ defined by equations $\alpha_{i} \cdot \xi=0$, $i=1, \ldots, n-m-r$. Then we can choose elements $\beta_{1}, \ldots, \beta_{m+r} \in \boldsymbol{Z}^{n}$ linearly independent of the $\alpha_{i}$. Since $k\left[X^{ \pm}\right] / I$ has transcendence degree $m$, the elements $X^{\beta_{i}}$ thereof will be algebraically dependent, hence we can find a nonzero element $a \in I \cap k\left[X^{\beta_{1}}, \ldots, X^{\beta_{m+r}}\right] . V_{\infty}$ lies in the spherical dual of the convex hull of $s(a)$, hence, in particular, in the union of the $n-2$-dimensional great spheres defined by the equations $\left(\alpha-\alpha^{\prime}\right) \cdot \xi=0$, where $\alpha$ and $\alpha^{\prime}$ range over pairs of distinct elements of $s(a)$.

Since each $\alpha-\alpha^{\prime}$ is linearly independent of $\alpha_{1}, \ldots, \alpha_{n-m-r}$, none of these spheres contains $\boldsymbol{S}_{0}$. Q.E.D.

Second assertion. Because rational hemispheres are dense in the space of all hemispheres, it will suffice to prove this claim for a rational hemisphere, defined by relations $\alpha_{1} \cdot \xi=\cdots=\alpha_{m-1} \cdot \xi=0, \alpha_{m} \cdot \xi \geqq 0\left(\alpha_{i} \in \boldsymbol{Z}^{n}\right)$.

If $X^{\alpha_{1}}, \ldots, X^{\alpha_{m}}$ are algebraically independent in $k\left[X^{ \pm}\right] / I$, then they generate a polynomial subring therein, and we can define on it a valuation $v$ with $v\left(X^{\alpha_{1}}\right)=\cdots=v\left(X^{\alpha_{m-1}}\right)=0, v\left(X^{\alpha_{m}}\right)=-1$. By [5, Chapter VI, Theorem 5'], this will extend to a valuation on all of $k\left[X^{ \pm}\right] / I$, possibly with larger value-group. We reduce this to a real-valued valuation as in $\S 4$, which we see will satisfy $v\left(X^{\alpha_{1}}\right)=\cdots=v\left(X^{\alpha_{m-1}}\right)=0, v\left(X^{\alpha_{m}}\right) \leqq 0$, and so the corresponding point of $V_{\infty}$ lies on our hemisphere.

If, on the other hand, the $X^{\alpha_{i}}$ are algebraically dependent over $k$, then $k\left[X^{ \pm}\right] / I$ is transcendental over the subring they generate, so by [5, Chapter VI, Theorem 6] we can find a valuation $v$ on this ring which is zero on that subring, but not on all $X_{i}$. This will yield a point $\xi \in V_{\infty}$ satisfying $\xi \cdot \alpha_{1}=\cdots=\xi \cdot \alpha_{m}=0$.

From the above result and Theorem 2, one can deduce that the same statements hold for $V_{\infty}^{(a)}$ - the key observation for obtaining the second assertion is that the intersection of a rational great $m-1$-sphere and a rational great hemisphere of dimension $n-m$, in general position, is a point with rational coordinate-ratios. One can deduce other properties of $V_{\infty}$ from the above theorem-for instance, it will contain an open subset of a rational great $m-1$-sphere. If the conjecture of our last section is true, so that $V_{\infty}$ is a finite union of spherical polytopes, this says that the maximum of their dimensions will be exactly $m-1$, and an interesting question is whether $V_{\infty}$ will be a union of polytopes all having dimension $m-1$.

A general field open for investigation is the geometric structure of $V_{\infty}-$ connectedness, homotopy and (co)homology groups, "vertex figures", etc.-and its relation to the properties of the variety $V$.

Appendix-the closure of an open subvariety. Theorem 1 of Chapter $1, \S 10$ of [3] says that a nonempty open subvariety $U$ of an irreducible variety $X$ over the complex numbers is dense in the "strong topology" on $X$-that induced by the 
topology of the complexes. The proof makes use of the local compactness of the complex field at one point, p. 114, line 5. But it may be modified to work over any nondiscrete algebraically closed topological field $k$ having the following continuityof-roots property also assumed in the proof given:

If $P$ is a monic polynomial over $k$, and $P^{\prime}$ another monic polynomial of the same degree whose coefficients are sufficiently near those of $P$, then every root of $P^{\prime}$ is near a root of $P$.

It is not hard to show that this holds for any algebraically closed field $k$ whose topology is defined by an absolute value.

To adapt the proof in [3]: we make no changes in pp. 111-113 (q.v.!), up to the last paragraph, except that we require the $g$ of p. 113, bottom, to satisfy $g\left(x_{2}\right)=$ $\cdots=g\left(x_{N}\right)=1$. We complete the argument as follows:

Let $h$ be any element of $\Gamma\left(X, o_{X}\right)$, and $P\left(X_{1}, \ldots, X_{n} ; h\right)$ its minimal polynomial over $k\left(X_{1}, \ldots, X_{n}\right)$, which, because $\Gamma\left(X, o_{X}\right)$ is integral over $k\left[X_{1}, \ldots, X_{n}\right]$, will have coefficients in that ring. On $X$, we have $P(\pi(z) ; h(z))=0$ identically. By our assumption on the topology of $k$, we see that when $i$ is large, so that $\pi\left(z^{(i)}\right)$ is near $\pi(x)$, the value $h\left(z^{(i)}\right)$, satisfying $P\left(\pi\left(z^{(i)}\right) ; h\left(z^{(i)}\right)\right)=0$, must be near a root of $P(\pi(x) ; t)=0$, i.e., one of the values $h\left(x_{1}\right), \ldots, h\left(x_{N}\right)$. Which one it is near could depend on $i$.

Choose $\alpha \in k$ not equal to any $h\left(x_{p}\right)-h\left(x_{q}\right)(p, q=1, \ldots, N)$. For $i$ sufficiently large, $h\left(z^{(i)}\right)$ will be near some $h\left(x_{p}\right)$, and $h(x+\alpha g)\left(z^{(i)}\right)$ will be near some $(h+\alpha g)\left(x_{q}\right)=h\left(x_{q}\right)+\alpha g\left(x_{q}\right)$. Since $g\left(z^{(i)}\right) \rightarrow 0$ (by choice of $z^{(i)}$, p. 113), they will also be near each other. But the only pair of $h\left(x_{p}\right)$ and $h\left(x_{q}\right)+\alpha g\left(x_{q}\right)$ that are equal is $h\left(x_{1}\right)=h\left(x_{1}\right)+\alpha g\left(x_{1}\right)$, by choice of $g$ and $\alpha$. So in fact, $h\left(z^{(i)}\right)$ must approach $h(x)$ for any $h$, which means that $z^{(i)} \rightarrow x$ in the function topology on $X$.

\section{REFERENCES}

1. Dniestr Notebook, unsolved problems in the theory of rings and modules, L. M. Andreichuk, Editor, 500 copies duplicated by the Acad. Sci. Moldavian SSR, June 1969, 6 kopeks. (Collection of 101 problems from the Kishinyev symposium on ring and module theory, 3-6 September, 1968. All but one of the other problems are for noncommutative or nonassociative rings. Russian.)

2. G. M. Bergman, A weak Nullstellensatz for valuations, Proc. Amer. Math. Soc. 28 (1971), 32-38.

3. D. Mumford, Introduction to algebraic geometry, Department of Mathematics, Harvard University, Cambridge, Mass., 1966 (preliminary version of first three chapters).

4. M. Raynaud, Modèles de Néron, C. R. Acad. Sci. Paris Sér. A-B 262 (1966), A345-A347. MR 33 \#2631.

5. O. Zariski and P. Samuel, Commutative algebra. Vol. 2, University Series in Higher Math., Van Nostrand, Princeton, N. J., 1960. MR 22 \#11006.

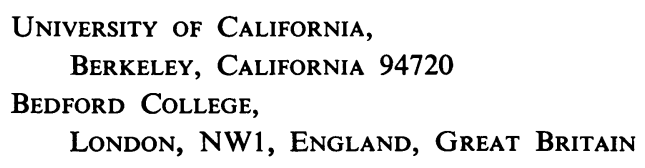

